\title{
Online Supplement for Quantifying causal effects of road network capacity expansions on traffic flow and density via a mixed model propensity score estimator
}

\author{
Daniel J. Graham ${ }^{\dagger}$, Emma J. McCoy ${ }^{\ddagger}$ and David A. Stephens ${ }^{\S}$ \\ Imperial College London \\ and \\ McGill University, Montreal
}

\section{A.1 Algorithm for estimation of a dose-response function us- ing the GPS}

1. Estimate a model for the conditional density of $D$ given $X$ with parameter vector $\theta$

$$
f_{D \mid X}\left(d_{i} \mid x_{i} ; \theta\right) .
$$

This can be achieved via estimation of a standard Generalized Linear Model (GLM), or Generalised Linear Mixed Model (GLMM) if random effects are assumed, with the choice of link function tailored to the assumed distribution for the conditional density.

2. Use $\widehat{\theta}$, with the assumed density function, to calculate the observed and unobserved GPSs: $\widehat{R}_{i}=f_{D \mid X}\left(d_{i} \mid x_{i} ; \widehat{\theta}\right)$ and $\widehat{R}_{d, i}=f_{D \mid X}\left(d \mid x_{i} ; \widehat{\theta}\right)$, for all $d$ of interest. For instance, assuming a normal density we calculate the observed GPS as

$$
\widehat{R}_{i}=f_{D \mid X}\left(d_{i} \mid x_{i}\right)=\frac{1}{\sqrt{2 \pi \widehat{\sigma}_{D \mid X}^{2}}} \exp \left(-\frac{1}{2 \widehat{\sigma}_{D \mid X}^{2}}\left(d_{i}-\widehat{d}_{i}\right)^{2}\right),
$$

where $\widehat{\sigma^{2}} D \mid X$, being an element of $\theta$, is an estimate of the conditional variance and $\widehat{d}_{i}$ is the fitted value from the regression model for $\mathbb{E}\left[D_{i} \mid X_{i}\right]$.. The unobserved GPS for dose $d, \widehat{R}_{d, i}$, is formed by letting $d_{i}=d$ in the definition of $\widehat{R}_{i}$.

3. Using values of $\widehat{R}_{d, i}$ across all $d$ of interest find a region of common support $\mathcal{C} \in \mathcal{D}$, and check that balancing holds for this region.

(a) Balancing - at present, there are a variety of different approaches proposed to assess balancing. Hirano and Imbens (2004), Mitnik (2007) and Flores et al. (2012) check

\footnotetext{
${ }^{\dagger}$ Corresponding author: Department of Civil Engineering, Imperial College London, London, SW7 2AZ, Email: d.j.graham@imperial.ac.uk

${ }^{\ddagger}$ Department of Mathematics, Imperial College London, London, SW7 2AZ, UK, Email: e.mccoy@imperial.ac.uk

${ }^{\S}$ Department of Mathematics and Statistics, McGill University, Montreal, H3A 2K6, Canada, Email: d.stephens@math.mcgill.ca
} 
for balancing by testing whether the confounding covariates have any predictive power in a regression model for the treatment given the GPS within discretized ranges of the treatment, while Imai and Van Dyk (2004) and Kluve et al. (2012) run separate regressions for each covariate with and without conditioning on the GPS. However assessed, the key concern for balancing is that the distribution of the treatment should not depend on the covariates within strata of the GPS.

For our application, in a similar manner to Hirano and Imbens (2004) and Flores et al. (2012), we regress the treatment on the covariates, the GPS, and a set of indicator variables corresponding to a discretization of the treatment into 11 strata. We then compare BIC values obtained from the linear regression models with and without covariates and conduct an F-test between the restricted (without covariates) and unrestricted models to test for balancing.

(b) Common support - there is currently no consensus on the procedure that should be used to identify and trim for common support, this is true even in the case of binary treatments (for discussion see Crump et al. 2009). Flores and Mitnik (2009), Neugebauer (2004) and Neugebauer and van der Laan (2005) show that common support is an important requirement and that violation of this assumption can have a large impact on the performance of causal estimators.

The common support requirement is generally more challenging to evaluate for multivalued and continuous treatments than for binary treatments because the property must hold over a range of doses. As Neugebauer (2004) and Neugebauer and van der Laan (2005) point out, since the possible set of continuous treatments is infinite they are by their nature likely to be deterministically assigned given the covariates.

Imbens (1999), Flores et al. (2012) and Kluve et al. (2012) take an approach based on discretisation of the treatment. They do this by defining strata of the treatment distribution, for instance 3 strata with cut-off at the 30th and 70th percentile of the distribution, and evaluate the GPS at the median point of each stratum. They do this for all units in the sample and compare the distribution of the GPSs evaluated at each strata median to the GPS distribution for the rest of the sample. A test for common support is then made graphically and the sample trimmed accordingly.

To identify the common support region we use a probability representation of the GPS which we refer to as the PGPS and denote it by $\pi$ rather than $R$. Given parameter estimates $\hat{\theta}$ for some assumed density, $f_{D \mid X}\left(d \mid x_{i} ; \hat{\theta}\right)$, and for some small $\delta$ defined around the dose of interest, $d$, we define

$$
\begin{aligned}
\hat{\pi}_{d, i} & =\operatorname{Pr}\left(d-\delta \leq D_{i} \leq d+\delta \mid X_{i} ; \hat{\theta}\right) \\
& =F_{D \mid X}\left(d+\delta \mid X_{i}, \hat{\theta}\right)-F_{D \mid X}\left(d-\delta \mid X_{i} ; \hat{\theta}\right) \\
& =\int_{d-\delta}^{d+\delta} f_{D \mid X}\left(t \mid x_{i} ; \hat{\theta}\right) d t \\
& \approx \mathbb{E}\left[I_{D_{i}}(d) \mid X_{i}=x\right] .
\end{aligned}
$$

Using the probability representation of the GPS we can approximate $\operatorname{Pr}\left(D_{i} \in\right.$ $\left.\mathcal{A}_{k} \mid X_{i}=x\right)$ for some chosen discretization of $\mathcal{D}$ into $k$ subsets $\mathcal{A}_{k} \subseteq \mathcal{D}$ where $\mathcal{A}_{k}=\left[d_{k}-\delta, d_{k}+\delta\right]$. To identify a region of common support $\mathcal{C} \subseteq \mathcal{D}$ we define a threshold, $t$ say, and find the set of values of $\mathcal{A}_{k}$ over which $\operatorname{Pr}\left(D_{i} \in \mathcal{A}_{k} \mid X_{i}=x\right) \geq t$ holds for all $\mathcal{A}_{k} \in \mathcal{C}$. The algorithm for evaluation of common support is therefore as follows: 
i. Using a regression model estimate the conditional density of $D_{i}$ given $X_{i}$ $f_{D_{i} \mid X_{i}}\left(d_{i} \mid X_{i} ; \theta\right)$ with parameter vector $\theta$.

ii. Using the estimated parameters $\hat{\theta}$, and an appropriate continuous density, take a discrete approximation to

$$
\hat{\pi}_{d, i}=\int_{d-\delta}^{d+\delta} f_{D \mid X}\left(t \mid x_{i}\right) d t
$$

for defined treatment levels of interest $d \in \mathcal{D}$ given choice of $\delta$ (as shown in equation (1)). If there are $k$ such treatment levels this yields an $n \times k$ matrix of PGPSs with row sums equal to $1.0^{1}$.

iii. Impose a rule for common support according to a minimum probability threshold. In this paper we impose $\hat{\pi}_{d, i} \geq 0.01$.

iv. Determine the range of treatments for which the common support rule holds for each $n$ rows.

v. Use row ranges to define regions of common support for one or more regions of treatment. We denote the number of observation in the common support region by $S$.

For our longitudinal simulation and application we calculate $\pi_{d, i t}$ which varies over time. We use a $\delta$ value of 0.00125 and calculate $\hat{\pi}_{d, i t}$ for treatment levels 1.0 through to 1.10 in increments of 0.0025 and form the PGPS matrix. To isolate the common support region we use the rule $\widehat{\pi}_{d, i t}>0.01$ for all $d$.

4. Estimate $\mathbb{E}\left(Y_{i} \mid D_{i}, \widehat{R}_{i}\right)$ using a flexible regression model with parameters $\alpha: m\left(y_{i} \mid d_{i}, \widehat{R}_{i} ; \alpha\right)$. This can be achieved through a flexible polynomial form or through use of flexible semiparametric regressions for local curve fitting (i.e. Ruppert et al. 2003). It is important to note that this model has no particular substantive interpretation, but is simply a device to adjust for confounding between $d_{i}$ and $x_{i}$ to estimate the dose response. Hirano and Imbens (2004) show that consistent estimates of the dose-response can be obtained via this route, but only if this conditional mean model is correctly specified. Provided $\mathbb{E}[Y \mid D, R]$ is bounded for each $(d, r)$, then a suitably flexible specification should consistently estimate the dose response function.

5. Average over predicted values from 3., evaluated at dose $d$, to obtain a point estimate of the expected response at $d: \widehat{\mu}(d)=S^{-1} \sum_{i=1}^{S} \mathbb{E}_{Y \mid D, R}\left[Y_{i}(d) \mid d, \widehat{R}_{d, i}, \widehat{\alpha}\right]$, and repeat for all doses of interest.

6. Use a single bootstrap re-sampling scheme over steps 1 to 5 for variance estimation.

\section{A.2 Proofs for properties of the FCGPS}

We refer to a GPS that conditions on confounding and non-confounding covariates as a Fully Conditional GPS (FCGPS). Let

$$
r^{*}\left(d, x_{i}, u_{i}\right)=f_{D \mid X, U}\left(d \mid x_{i}, u_{i}\right),
$$

\footnotetext{
${ }^{1}$ The choice of $\delta$ determines the number of $k$ required to achieve full coverage of the dose range of interest. As $\delta$ increases the conditional probabilities will grow in magnitude.
} 
represent a value from the conditional density of $D_{i}=d$ given both $X_{i}$ and $U_{i}$, which can be used to form the FCGPS random quantities $R_{i}^{*}=r^{*}\left(D_{i}, X_{i}, U_{i}\right)$ and $R_{d, i}^{*}=r^{*}\left(d, X_{i}, U_{i}\right)$ for fixed $d$. There are five properties of the FCGPS relevant for dose-response estimation that we show here.

\section{i. The FCGPS has a balancing property such that.}

$$
I_{D_{i}}(d) \Perp X_{i}, U_{i} \mid r^{*}\left(d, X_{i}, U_{i}\right) .
$$

This follows because $r^{*}\left(d, X_{i}, U_{i}\right)$ is a function of $X_{i}$ and $U_{i}$ only, so conditioning on $X_{i}$ and $U_{i}$ adds no additional information

$$
\mathbb{E}\left[I_{D_{i}}(d) \mid X_{i}, U_{i}, r^{*}\left(d, X_{i}, U_{i}\right)\right]=\mathbb{E}\left[I_{D_{i}}(d) \mid r^{*}\left(d, X_{i}, U_{i}\right)\right] .
$$

\section{ii. Ignorability can be established conditional on the FCGPS.}

Theorem 1. (Ignorability given the FCGPS). If assignment to the treatment is ignorable given pre-treatment characteristics $X_{i}$, then for all $D_{i}=d$

$$
Y_{i}(d) \Perp I_{D_{i}}(d) \mid r^{*}\left(d, X_{i}, U_{i}\right) .
$$

Proof: To prove that $Y_{i}(d)$ is conditionally independent of $D_{i}=d$, given the FCGPS and the assumption of ignorability, we can modify the analogous proof for the GPS given by Hirano and Imbens (2004) by integrating over $U_{i}$ as well as $X_{i}$. The proof shows that $f_{D \mid R^{*}, Y}\left(d \mid r^{*}\left(d, x_{i}, u_{i}\right), Y_{i}(d)\right)=f_{D \mid R^{*}}\left(d \mid r^{*}\left(d, x_{i}, u_{i}\right)\right)$.

Let $\mathcal{X}$ and $\mathcal{U}$ be the sample space in which covariates $X_{i}$ and $U_{i}$ lie, then

$$
\begin{aligned}
f_{D \mid R^{*}} & \left(d \mid r^{*}\left(d, x_{i}, u_{i}\right)\right)=\int_{\mathcal{X}} \int_{\mathcal{U}} f_{D, X, U \mid R^{*}}\left(d, x_{i}, u_{i} \mid r^{*}\left(d, x_{i}, u_{i}\right)\right) d x_{i} d u_{i} \\
& =\int_{\mathcal{X}} \int_{\mathcal{U}} f_{D \mid X, U, R^{*}}\left(d \mid x_{i}, u_{i}, r^{*}\left(d, x_{i}, u_{i}\right)\right) f_{X, U \mid R^{*}}\left(x_{i}, u_{i} \mid r^{*}\left(d, x_{i}, u_{i}\right)\right) d x_{i} d u_{i} \\
& =\int_{\mathcal{X}} \int_{\mathcal{U}} f_{D \mid X, U}\left(d \mid x_{i}, u_{i}\right) f_{X, U \mid R^{*}}\left(x_{i}, u_{i} \mid r^{*}\left(d, x_{i}, u_{i}\right)\right) d x_{i} d u_{i} \\
& =\int_{\mathcal{X}} \int_{\mathcal{U}} r^{*}\left(d, x_{i}, u_{i}\right) f_{X, U \mid R^{*}}\left(x_{i}, u_{i} \mid r^{*}\left(d, x_{i}, u_{i}\right)\right) d x_{i} d u_{i}, \\
& =r^{*}\left(d, x_{i}, u_{i}\right)=f_{D \mid X, U}\left(d \mid x_{i}, u_{i}\right),
\end{aligned}
$$

Thus, $f_{D \mid X, U, R^{*}}\left(d \mid r^{*}\left(d, x_{i}, u_{i}\right)\right)=f_{D \mid X, U}\left(d \mid x_{i}, u_{i}\right)=r^{*}\left(d, x_{i}, u_{i}\right)$. Similarly,

$$
\begin{aligned}
f_{D \mid R^{*}, Y} & \left(d \mid r^{*}\left(d, x_{i}, u_{i}\right), Y_{i}(d)\right)=\int_{\mathcal{X}} \int_{\mathcal{U}} f_{D, X, U \mid R^{*}, Y}\left(d, x_{i}, u_{i} \mid r^{*}\left(d, x_{i}, u_{i}\right), Y_{i}(d)\right) d x_{i} d u_{i} \\
& =\int_{\mathcal{X}} \int_{\mathcal{U}} f_{D \mid X, U, R^{*}, Y}\left(d \mid x_{i}, u_{i}, r^{*}\left(d, x_{i}, u_{i}\right), Y_{i}(d)\right) f_{X, U \mid R^{*}, Y}\left(x_{i}, u_{i} \mid r^{*}\left(d, x_{i}, u_{i}\right), Y_{i}(d)\right) d x_{i} d u_{i} \\
& =\int_{\mathcal{X}} \int_{\mathcal{U}} f_{D \mid X, U}\left(d \mid x_{i}, u_{i}\right) f_{X, U \mid R^{*}, Y}\left(x_{i}, u_{i} \mid r^{*}\left(d, x_{i}, u_{i}\right), Y_{i}(d)\right) d x_{i} d u_{i} \\
& =\int_{\mathcal{X}} \int_{\mathcal{U}} r^{*}\left(d, x_{i}, u_{i}\right) f_{X, U \mid R^{*}, Y}\left(x_{i}, u_{i} \mid r^{*}\left(d, x_{i}, u_{i}\right), Y_{i}(d)\right) d x_{i} d u_{i} \\
& =r^{*}\left(d, x_{i}, u_{i}\right)=f_{D \mid X, U}\left(d \mid x_{i}, u_{i}\right) .
\end{aligned}
$$

Therefore, for all $d, f_{D \mid R^{*}}\left(d \mid r^{*}\left(d, x_{i}, u_{i}\right)\right)=f_{D \mid R^{*}, Y}\left(d \mid r^{*}\left(d, x_{i}, u_{i}\right), Y_{i}(d)\right)$ and we have ignorability given $R_{d, i}^{*}=r^{*}\left(d, X_{i}, U_{i}\right)$. 
iii. Conditioning on the FCGPS eliminates biases associated with differences in the confounding covariates $X_{i}$.

This follows from the assumption of ignorability and the balancing property of the FCGPS.

Theorem 2. (Bias removal of the FCGPS). Suppose that assignment to the treatment is ignorable given pre-treatment characteristics $X_{i}$. Then define

$$
\beta\left(d, r^{*}\left(d, X_{i}, U_{i}\right)\right)=\mathbb{E}\left[Y_{i}(d) \mid I_{D_{i}}(d), r^{*}\left(d, X_{i}, U_{i}\right)\right],
$$

as the conditional mean of potential outcome, given the treatment level $D=d$ and the $F C G P S$, for fixed $d \in \mathcal{D}$. The expectation of $\beta\left(d, r^{*}\left(d, X_{i}, U_{i}\right)\right)$ over $X_{i}$ gives the doseresponse function

$$
\mu(d)=\mathbb{E}\left[Y_{i}(d)\right]=\mathbb{E}_{X}\left[\mathbb{E}\left[Y(d) \mid X_{i}\right]\right] .
$$

Proof: The proof involves a minor modification to the analogous result for the GPS given in Hirano and Imbens (2004). By conditional probability and theorem (1) above we have

$$
\begin{aligned}
f_{Y(d) \mid D, R^{*}}\left(y_{i}(d) \mid d, r^{*}\left(d, x_{i}, x_{i}\right)\right) & =\frac{f_{Y(d) \mid R^{*}}\left(y_{i}(d) \mid r^{*}\left(d, x_{i}, u_{i}\right)\right) f_{D \mid Y(d), R^{*}}\left(d \mid y_{i}(d), r^{*}\left(d, x_{i}, u_{i}\right)\right)}{f_{D \mid R^{*}}\left(d \mid r^{*}\left(d, x_{i}, u_{i}\right)\right)} \\
& =\frac{f_{Y(d) \mid R^{*}}\left(y_{i}(d) \mid r^{*}\left(d, x_{i}, u_{i}\right)\right) f_{D \mid R^{*}}\left(d \mid r^{*}\left(d, x_{i}, u_{i}\right)\right)}{f_{D \mid R^{*}}\left(d \mid r^{*}\left(d, x_{i}, u_{i}\right)\right)} \\
& =f_{Y(d) \mid R^{*}}\left(y_{i}(d) \mid r^{*}\left(d, x_{i}, u_{i}\right)\right),
\end{aligned}
$$

and so by iterated expectations,

$$
\begin{aligned}
\mu(d) & =\mathbb{E}\left[Y_{i}(d)\right]=\mathbb{E}_{X}\left[\mathbb{E}\left[Y(d) \mid X_{i}\right]\right]=\mathbb{E}_{X, U}\left[\mathbb{E}\left[Y_{i}(d) \mid I_{D_{i}}(d), r^{*}\left(d, X_{i}, U_{i}\right)\right]\right] \\
& =\mathbb{E}_{X, U}\left[\mathbb{E}\left[Y_{i} \mid I_{D_{i}}(d), r^{*}\left(d, X_{i}, U_{i}\right)\right]\right]
\end{aligned}
$$

iv. Estimates of the dose-response based on the true GPS will be at least as efficient as than those based on the FCGPS.

This property could be shown in several different ways. Here we simply make the general point that the variance of the conditional expectation values used to form the dose-response are higher for the FCGPS than the GPS. Under full conditioning we have

$$
\begin{aligned}
\operatorname{Var}\left(\mathbb{E}\left[Y_{i}(d) \mid X_{i}, U_{i}\right]\right) & =\operatorname{Var}\left(\mathbb{E}\left[\mathbb{E}\left[Y_{i}(d) \mid X_{i}, U_{i}\right] \mid U\right]\right)+\mathbb{E}\left[\operatorname{Var}\left(\mathbb{E}\left[Y_{i}(d) \mid X_{i}, U_{i}\right] \mid U\right)\right] \\
& =\operatorname{Var}\left(\mathbb{E}\left[Y_{i}(d) \mid X_{i}\right]\right)+\mathbb{E}\left[\operatorname{Var}\left(\mathbb{E}\left[Y_{i}(d) \mid X_{i}, U_{i}\right] \mid U\right)\right]
\end{aligned}
$$

and since $\mathbb{E}\left[\operatorname{Var}\left(\mathbb{E}\left[Y_{i}(d) \mid X_{i}, U_{i}\right] \mid U\right)\right] \geq 0$, then $\operatorname{Var}\left(\mathbb{E}\left[Y_{i}(d) \mid X_{i}, U_{i}\right)\right] \geq \operatorname{Var}\left(\mathbb{E}\left[Y_{i}(d) \mid X_{i}\right]\right)$.

v. The common support region established using the GPS will be at least as large as that established using the FCGPS.

The GPS, $r\left(d, X_{i}\right)$, and the FCGPS, $r\left(d, X_{i}, U_{i}\right)$, are random variables defined with respect to the conditional densities $f_{D \mid X}\left(d \mid x_{i}\right)$ and $f_{D \mid X}\left(d \mid x_{i}, u_{i}\right)$ respectively. To calculate values of the GPS and FCGPS we use observed data to model conditional means: $\mathbb{E}\left[D_{i} \mid X_{i}\right]$ and $\mathbb{E}\left[D_{i} \mid X_{i}, U_{i}\right]$; and conditional variances: $\operatorname{Var}\left(D_{i} \mid X_{i}\right)$ and $\operatorname{Var}\left(D_{i} \mid X_{i}, U_{i}\right)$. From the identity for conditional variance

$$
\mathbb{E}\left[\operatorname{Var}\left(D_{i} \mid X_{i}\right)\right]=\operatorname{Var}\left(D_{i}\right)-\operatorname{Var}\left(\mathbb{E}\left[D_{i} \mid X_{i}\right]\right), \text { and }
$$




$$
\begin{gathered}
\mathbb{E}\left[\operatorname{Var}\left(D_{i} \mid X_{i}, U_{i}\right)\right]=\operatorname{Var}\left(D_{i}\right)-\operatorname{Var}\left(\mathbb{E}\left[D_{i} \mid X_{i}, U_{i}\right]\right), \text { but } \\
\operatorname{Var}\left(\mathbb{E}\left[D_{i} \mid X_{i}, U_{i}\right]\right)=\operatorname{Var}\left(\mathbb{E}\left[\mathbb{E}\left[D_{i} \mid X_{i}, U_{i}\right] \mid U\right]\right)+\mathbb{E}\left[\operatorname{Var}\left(\mathbb{E}\left[D_{i} \mid X_{i}, U_{i}\right] \mid U\right)\right] \\
=\operatorname{Var}\left(\mathbb{E}\left[D_{i} \mid X_{i}\right]\right)+\mathbb{E}\left[\operatorname{Var}\left(\mathbb{E}\left[D_{i} \mid X_{i}, U_{i}\right] \mid U\right)\right]
\end{gathered}
$$

and since $\mathbb{E}\left[\operatorname{Var}\left(\mathbb{E}\left[D_{i} \mid X_{i}, U_{i}\right] \mid U\right)\right] \geq 0$, then $\operatorname{Var}\left(\mathbb{E}\left[D_{i} \mid X_{i}, U_{i}\right]\right) \geq \operatorname{Var}\left(\mathbb{E}\left[D_{i} \mid X_{i}\right]\right)$, and thus

$$
\mathbb{E}\left[\operatorname{Var}\left(D_{i} \mid X_{i}, U_{i}\right)\right] \leq \mathbb{E}\left[\operatorname{Var}\left(D_{i} \mid X_{i}\right)\right]
$$

or if the variances are constant, as we assume in PS construction, $\operatorname{Var}\left(D_{i} \mid X_{i}, U_{i}\right) \leq$ $\operatorname{Var}\left(D_{i} \mid X_{i}\right)$. Thus, the FCGPS values are drawn from a conditional density that has smaller variance than the conditional density from which the GPS values are drawn. Since this implies that at any dose $d$ the FCGPS conditional density will have greater mass concentrated over a smaller range of units, the common support region over $\mathcal{D}$ established using the GPS will be at least as large as that using the FCGPS.

With more extensive conditioning under the FCGPS, estimates of the dose response function remain unbiased, although potentially less efficient. For a discussion of bias-variance trade-off in the context of casual models see Hernán and Robins (2012).

It is important to note that covariates unrelated to the outcome but correlated with the treatment have the characteristics of instrumental variables (IVs), and that conditioning on IVs can increase unmeasured confounding bias (e.g. Pearl 2010). Myers et al. (2011) analyse this problem and find that conditioning on a perfect IV, or near perfect IV, may give rise to larger bias than the unconditional model, but in most of their scenarios the error was small. They conclude that minimizing unmeasured confounding should take priority over concern about IVs.

\section{A.3 Data}

The data are described in detail in the Texas Transportation Institute (TTI) documentation, available at http://mobility.tamu.edu/ums/, and also in recent studies by Hymel et al. (2010) and Melo et al. (2013). Some non-transport related covariates required for our analysis are not included in the TTI data and have instead been taken from the metropolitan area statistics published by the US Bureau of Economic Affairs (BEA), available at http://www . bea.gov/regional/.

Our response variable is annual proportional change in aggregate urban traffic volume, with volume measured by annual vehicle miles travelled (vmt) per annum in each city $i$ : $y_{i t}=$ $v m t_{i t} / v m t_{i t-1}$. Note that the TTI data are measured at each year end so this variable describes the proportional change in volume that occurred over year $t$. Our treatment variable is a measure of the proportional change in urban road lane miles (lms) in each year: $d_{i t}=$ $l m s_{i t} / l m s_{i t-1}$.

\section{A.3.1 Confounding covariates}

The potential outcomes framework requires that confounding covariates must be 'pre-treatment', that is they must have values that are fixed before the relevant treatment for each interval 
is measured. For this reason we measure all confounders in the year immediately prior to the treatment. To model time-varying confounders we use the available longitudinal data to construct the following covariates:

1. Lagged-response - the relationship between capacity assignment and traffic volume is thought to be bi-directional and previous values of the response may therefore be relevant for future values.

2. Congestion (annual hours of delay per vmt) - additional road capacity may be assigned to combat congestion which may itself have a bearing on growth in vmt. The TTI data provide a measures of total annual delay for each city which uses highly disaggregate speed-flow observations to approximate the total time lost due to differences between congested and free-flow speeds. Our congestion covariates is measured by total delay per vmt.

3. Traffic volume (vmt) - we expect capacity to be assigned in relation to traffic volumes and we allow for the possibility that the initial scale of traffic can affect its proportional growth.

4. Network scale $(l m s)$ - proportional growth in capacity depends on initial network scale, which may also affect growth in volume.

5. Network composition (freeway lms / arterial lms) - the data distinguish between freeway and arterial lane miles. Freeways are express routes carrying a high proportion of long distance traffic, while arterials provide local connectivity with a higher proportion of short distance trips. We expect the relative mix of road types to be relevant for vmt growth and also for decisions on allocation of new capacity. We take a simple ratio of lane miles in each road type as the covariate.

6. Traffic composition (freeway vmt / arterial vmt) - similarly, we measure the ratio of traffic on arterial to freeway to adjust for traffic mix by route type.

7. Mode share (annual miles travelled by public transport) - the extent of public transport (PT) patronage in the city may affect both assignment and response, so we include a measure of annual PT patronage measured in terms of PT passenger miles travelled.

8. Productivity (average metropolitan wage rate in \$'s per annum) - capacity expansions are often allocated to stimulate or accommodate economic productivity, but because transport is a derived demand, productivity in turn has a direct relationship with vmt. We include a measure of the metropolitan wage rate to proxy for productivity.

9. Economic structure (\% metropolitan jobs in manufacturing) - we expect different types of regional economy to have different vmt and different capacity requirements. To adjust for this potential source of confounding we represent metropolitan economic structure through the share of manufacturing jobs.

10. Employment and population distribution and growth - capacity assignment is likely to be influenced by the relative distribution and growth of metropolitan employment and population. We construct covariates measuring the levels (i.e. number of people) and proportional growth of employment and population.

11. Personal income (personal income in \$'s per annum) - we include a measure of metropolitan personal income in dollars per annum to reflect the standard of living of the city. 
We expect income to be a confounder for reasons similar to those given in relation to productivity.

12. State fuel price (\$per gallon) - the state fuel price will influence vmt growth but may also be a significant factor in capacity allocation, particularly because it will in part determine the revenue available to the transport planning authority.

Note that the data for confounders 8 to 12 are sourced from the BEA and relate to Standard Metropolitan Statistical areas, which the TTI city boundaries nest within.

\section{A.3.2 Summary statistics}

Table A1: Case study data summary statistics $(n=2474)$

\begin{tabular}{l|cccc}
\hline & min & mean & max & s.d. \\
\hline Response & 0.542 & 1.030 & 2.037 & 0.041 \\
Treatment & 1.000 & 1.019 & 1.099 & 0.016 \\
Congestion & 0.008 & 0.140 & 0.595 & 0.081 \\
Traffic volume (000s) & 590.000 & 23752.708 & 260775.000 & 33320.463 \\
Network scale & 185.000 & 3215.447 & 27020.000 & 3976.809 \\
Network composition & 0.843 & 3.543 & 17.778 & 2.203 \\
Traffic composition & 0.353 & 1.482 & 14.521 & 1.298 \\
Mode share (million) & 0.181 & 411.110 & 21699.268 & 1829.315 \\
Productivity & 23139.380 & 39193.966 & 92791.820 & 7631.515 \\
Economic structure & 0.012 & 0.106 & 0.316 & 0.051 \\
Population (000s) & 116.566 & 1917.068 & 19069.796 & 2621.578 \\
Employment (000s) & 39.429 & 1101.052 & 11023.744 & 1489.525 \\
Population growth & -0.033 & 0.014 & 0.123 & 0.013 \\
Employment growth & -0.085 & 0.020 & 0.127 & 0.023 \\
Personal income & 11931.589 & 33771.609 & 81987.926 & 7924.634 \\
State fuel price & 1.207 & 1.999 & 3.922 & 0.548 \\
\hline
\end{tabular}

Table A2: Summary statistics for observations in the common support region $(S=1353)$

\begin{tabular}{lcccc}
\hline & min & mean & max & s.d. \\
\hline Response & 0.857 & 1.029 & 2.037 & 0.045 \\
Treatment & 1.000 & 1.016 & 1.075 & 0.012 \\
Congestion & 0.008 & 0.137 & 0.594 & 0.082 \\
Traffic volume & 625.000 & 25465.785 & 260775.000 & 31805.662 \\
Network scale & 185.000 & 3516.916 & 26905.000 & 3987.799 \\
Network composition & 0.843 & 3.408 & 17.778 & 2.186 \\
Traffic composition & 0.353 & 1.369 & 14.521 & 1.244 \\
Mode share & 1.401 & 482.310 & 19831.519 & 2038.681 \\
Productivity & 23139.380 & 39327.511 & 92791.820 & 7566.722 \\
Economic structure & 0.013 & 0.111 & 0.316 & 0.051 \\
Population (000s) & 118.164 & 1948.495 & 18825.633 & 2569.997 \\
Employment (000s) & 42.213 & 1116.865 & 10663.921 & 1441.278 \\
Population growth & -0.033 & 0.012 & 0.068 & 0.011 \\
Employment growth & -0.085 & 0.019 & 0.105 & 0.021 \\
Personal income & 12256.477 & 33824.855 & 75438.509 & 7635.594 \\
State fuel price & 1.259 & 1.920 & 3.922 & 0.449 \\
\hline
\end{tabular}




\section{A.4 Additional results}

\section{A.4.1 GPS models}

We consider two additional GPS specifications.

1. Adjustment for measured confounders (GPS)

$$
D_{i t} \mid X_{i t} \sim \mathcal{N}\left(\theta_{1}+X_{i t}^{\top} \theta_{2}, \sigma_{1}^{2}\right) .
$$

The model adjusts for confounding from measured time-varying covariates only. Taking the fitted values and the dispersion parameter from the model as estimates of density moments, we construct the GPSs values using the approach outlined in the paper.

2. Multivariate GPS (MGPS)

$$
D_{i t} \mid X_{i t}, D_{i t-1}, Y_{i t-1} \sim \mathcal{N}\left(\theta_{1}+\theta_{2} D_{i t-1}+\theta_{3} Y_{i t-1}+X_{i t}^{\top} \theta_{4}, \sigma_{2}^{2}\right) .
$$

The MGPS is explained in the main body of the paper. Here we adopt a version that includes lags of order one in the treatment and response variables in addition to measured confounders.

Full results from these models are given in tables A3 to A5.

Table A3: REML Gaussian penalised spline model results for the conditional density of the treatment given covariates.

\begin{tabular}{lcccc}
\hline & \multicolumn{3}{c}{ Approx sig smooth terms: } \\
& $\mathrm{F}$ & $\mathrm{p}$-value & $\mathrm{F}$ & $\mathrm{p}$-value \\
\hline lag response & 14.712 & $<2 \mathrm{e}-16$ & 2.729 & 0.022 \\
lag treatment & - & - & 118.341 & $<2 \mathrm{e}-16$ \\
congestion & 0.967 & $3.26 \mathrm{e}-01$ & 0.348 & 0.740 \\
traffic flow & 10.303 & $4.84 \mathrm{e}-06$ & 14.553 & 0.000 \\
network scale & 9.138 & $2.75 \mathrm{e}-09$ & 13.782 & 0.000 \\
network composition & 5.779 & $1.31 \mathrm{e}-07$ & 0.599 & 0.582 \\
flow composition & 7.356 & $2.97 \mathrm{e}-10$ & 1.521 & 0.179 \\
economic structure & 8.521 & $1.36 \mathrm{e}-11$ & 4.014 & 0.011 \\
productivity & 1.154 & 0.329 & 0.311 & 0.720 \\
mode share & 6.924 & $8.71 \mathrm{e}-09$ & 3.430 & 0.012 \\
employment & 9.651 & $2.50 \mathrm{e}-11$ & 7.659 & 0.000 \\
fuel price & 14.009 & $4.61 \mathrm{e}-07$ & 14.697 & 0.000 \\
personal income & 2.856 & 0.004 & 1.272 & 0.277 \\
population growth & 10.990 & $4.46 \mathrm{e}-11$ & 5.966 & 0.000 \\
year & 8.152 & $6.00 \mathrm{e}-11$ & 1.949 & 0.101 \\
& & & & \\
deviance & $1.61 \mathrm{e}-04$ & & & \\
& & & -14895 & \\
BIC & -14091 & & & \\
& & & & \\
$n$ & 2474 & & & \\
\hline
\end{tabular}


Table A4: Results from a penalised spline models for the conditional mean of response given treatment and the GPS.

\begin{tabular}{lcccc}
\hline & \multicolumn{4}{c}{ Approx sig smooth terms: } \\
& \multicolumn{2}{c}{ GPS } & \multicolumn{2}{c}{ MGPS } \\
& $\mathrm{F}$ & $\mathrm{p}$-value & $\mathrm{F}$ & $\mathrm{p}$-value \\
\hline$D$ & 12.048 & 0.000 & 17.362 & $3.29 \mathrm{e}-14$ \\
$\hat{R}$ & 1.696 & $1.51 \mathrm{e}-01$ & 0.717 & 0.629 \\
& & & & \\
BIC & -6151 & & -5743 & \\
$S$ & 1779 & & 1685 & \\
\hline
\end{tabular}

Table A5: GPS estimates of the dose-response functions and associated ATEs \& elasticities with bootstrapped standard errors.

\begin{tabular}{c|cccccc|cccccc}
\hline dose & \multicolumn{9}{c}{ GPS } & \multicolumn{1}{c}{ MGPS } & \multicolumn{4}{c}{ MG. } \\
& $\widehat{\mu}(d)$ & S.E. & $\widehat{\tau}(d)$ & S.E. & elas & S.E. & $\widehat{\mu}(d)$ & S.E. & $\widehat{\tau}(d)$ & S.E. & elas & S.E. \\
\hline 1.000 & 1.015 & $(0.003)$ & - & - & - & - & 1.009 & $(0.003)$ & - & - & - & - \\
1.005 & 1.020 & $(0.002)$ & 0.005 & $(0.002)$ & 1.019 & $(0.468)$ & 1.018 & $(0.002)$ & 0.009 & $(0.003)$ & 1.782 & $(0.557)$ \\
1.010 & 1.026 & $(0.002)$ & 0.011 & $(0.003)$ & 1.072 & $(0.287)$ & 1.026 & $(0.002)$ & 0.017 & $(0.003)$ & 1.675 & $(0.286)$ \\
1.015 & 1.031 & $(0.003)$ & 0.016 & $(0.004)$ & 1.056 & $(0.279)$ & 1.032 & $(0.003)$ & 0.023 & $(0.005)$ & 1.538 & $(0.310)$ \\
1.020 & 1.034 & $(0.003)$ & 0.019 & $(0.004)$ & 0.949 & $(0.197)$ & 1.035 & $(0.003)$ & 0.026 & $(0.004)$ & 1.286 & $(0.203)$ \\
1.025 & 1.035 & $(0.002)$ & 0.020 & $(0.003)$ & 0.784 & $(0.130)$ & 1.035 & $(0.003)$ & 0.026 & $(0.004)$ & 1.029 & $(0.144)$ \\
1.030 & 1.036 & $(0.003)$ & 0.020 & $(0.004)$ & 0.680 & $(0.126)$ & 1.036 & $(0.003)$ & 0.026 & $(0.004)$ & 0.879 & $(0.130)$ \\
1.035 & 1.038 & $(0.003)$ & 0.023 & $(0.004)$ & 0.653 & $(0.109)$ & 1.038 & $(0.004)$ & 0.028 & $(0.004)$ & 0.809 & $(0.112)$ \\
1.040 & 1.041 & $(0.004)$ & 0.025 & $(0.004)$ & 0.632 & $(0.103)$ & 1.041 & $(0.005)$ & 0.031 & $(0.005)$ & 0.787 & $(0.126)$ \\
1.045 & 1.043 & $(0.004)$ & 0.027 & $(0.005)$ & 0.606 & $(0.107)$ & 1.045 & $(0.006)$ & 0.036 & $(0.007)$ & 0.789 & $(0.153)$ \\
1.050 & 1.044 & $(0.006)$ & 0.029 & $(0.007)$ & 0.582 & $(0.138)$ & 1.049 & $(0.011)$ & 0.039 & $(0.011)$ & 0.786 & $(0.230)$ \\
\hline
\end{tabular}

\section{A.4.2 Panel outcome regression models}

Below we provide results corresponding to the following panel outcome regressions (OR)

1. Pooled OLS (POLS) without covariates: $y_{i t}=\beta_{0}+\beta_{1} d_{i t}+\beta_{2} d_{i t}^{2}+\varepsilon_{i t}$.

2. Pooled OLS (POLS) with covariates: $y_{i t}=\beta_{0}+\beta_{1} d_{i t}+\beta_{2} d_{i t}^{2}+x_{i t}^{\top} \beta_{3}+\varepsilon_{i t}$.

3. Random effects (RE): $y_{i t}=\beta_{0 i}+\beta_{1} d_{i t}+\beta_{2} d_{i t}^{2}+x_{i t}^{\top} \beta_{3}+\varepsilon_{i t}$, with $\beta_{0 i} \sim \mathcal{N}\left(0, \sigma_{\beta_{0}}^{2}\right)$.

4. Fixed effects (FE): $y_{i t}=\beta_{0 i}+\beta_{1} d_{i t}+\beta_{2} d_{i t}^{2}+x_{i t}^{\top} \beta_{3}+\varepsilon_{i t}$, with $\beta_{0 i}$ fixed.

5. First difference (FD):

$$
\left(y_{i t}-y_{i t-1}\right)=\beta_{1}\left(d_{i t}-d_{i t-1}\right)+\beta_{2}\left(d_{i t}^{2}-d_{i t-1}^{2}\right)+\left(x_{i t}-x_{i t-1}\right)^{\top} \beta_{3}+\left(\varepsilon_{i t}-\varepsilon_{i t-1}\right) .
$$

6. Dynamic-panel Generalized Method of Moments (DPGMM):

$y_{i t}=\beta_{0 i}+\beta_{1} d_{i t}+\beta_{2} d_{i t}^{2}+x_{i t}^{\top} \beta_{3}+\varepsilon_{i t}$, with $\beta_{0 i} \sim \mathcal{N}\left(0, \sigma_{\beta_{0}}^{2}\right)$ or fixed. The lagged response is assumed endogenous.

The POLS models and the FD models are estimated using Ordinary Least Squares (OLS). The RE model is estimated using Generalized Least Squares (GLS). The FE model is estimated using the 'within-estimator', such that for model $y_{i t}=x_{i t}^{\top} \beta+\varepsilon_{i t}$ the within transformation, $\left(y_{i t}-\bar{y}_{i}\right)=\left(x_{i t}-\bar{x}_{i}\right)^{\top} \beta+\left(\varepsilon_{i t}-\bar{\varepsilon}_{i}\right)$, is used for parameter estimation, where $\bar{x}_{i}$ is the average vale of the covariates for each unit over time. The DPGMM estimator is estimated using 
Generalized Methods of Moments (GMM). The key issues with this dynamic model is that the unobserved individual effect $\beta_{0 i}$ will be correlated with the lagged response variable, and this can be addressed via instrumental variables. We use the system form of GMM estimation which compromises both first-differenced and levels equations with the time series nature of the data being used to derive a set of instruments from lagged levels (or differences) which are assumed correlated with the differenced (or level) endogenous covariates but orthogonal to the errors. Detail on the panel estimators can be found in standard textbooks such as Baltagi (2002), Cameron and Trivedi (2005), and Wooldridge (2010).

Full results from these models are given in table A6 to A9.

Table A6: Panel outcome regression model estimates.

\begin{tabular}{|c|c|c|c|c|c|c|c|c|c|c|c|c|}
\hline & \multicolumn{2}{|c|}{ POLS } & \multicolumn{2}{|c|}{ POLS } & \multicolumn{2}{|c|}{$\mathrm{RE}$} & \multicolumn{2}{|c|}{$\overline{\mathrm{FE}}$} & \multicolumn{2}{|c|}{ FD } & \multicolumn{2}{|c|}{ GMM } \\
\hline & Est. & S.E. & Est. & S.E. & Est. & S.E. & Est. & S.E. & Est. & S.E. & Est. & S.E. \\
\hline (Intercept) & -11.691 & 2.186 & -3.742 & 2.162 & -3.821 & 2.173 & - & - & 0.001 & 0.001 & - & - \\
\hline$D$ & 23.971 & 4.252 & 8.796 & 4.202 & 8.944 & 4.224 & 9.506 & 4.198 & 5.731 & 3.032 & 13.126 & 0.045 \\
\hline$D^{2}$ & -11.270 & 2.067 & -3.976 & 2.041 & -4.046 & 2.051 & -4.333 & 2.038 & -2.519 & 1.470 & -5.942 & 4.082 \\
\hline congestion & - & - & -0.005 & 0.002 & -0.005 & 0.002 & 0.019 & 0.005 & 0.041 & 0.008 & 0.056 & 1.980 \\
\hline traffic flow & - & - & -0.031 & 0.005 & -0.036 & 0.006 & -0.267 & 0.015 & -1.161 & 0.024 & -0.915 & 0.014 \\
\hline network scale & - & - & 0.031 & 0.006 & 0.035 & 0.006 & 0.195 & 0.016 & 0.653 & 0.039 & 0.729 & 0.211 \\
\hline network composition & - & - & 0.009 & 0.004 & 0.009 & 0.004 & -0.051 & 0.009 & -0.148 & 0.021 & -0.087 & 0.160 \\
\hline flow composition & - & - & -0.013 & 0.003 & -0.014 & 0.004 & -0.029 & 0.006 & -0.125 & 0.013 & -0.157 & 0.046 \\
\hline economic structure & - & - & -0.006 & 0.002 & -0.007 & 0.002 & -0.028 & 0.007 & -0.017 & 0.015 & 0.019 & 0.091 \\
\hline productivity & - & - & -0.023 & 0.012 & -0.024 & 0.012 & -0.026 & 0.023 & 0.012 & 0.048 & 0.009 & 0.020 \\
\hline mode share & - & - & 0.002 & 0.001 & 0.002 & 0.001 & -0.001 & 0.002 & -0.002 & 0.004 & -0.017 & 0.051 \\
\hline employment & - & - & 0.000 & 0.002 & 0.001 & 0.002 & 0.076 & 0.013 & 0.259 & 0.037 & -0.033 & 0.007 \\
\hline fuel price & - & - & -0.027 & 0.004 & -0.027 & 0.004 & -0.039 & 0.004 & -0.016 & 0.005 & -0.025 & 0.053 \\
\hline personal income & - & - & 0.024 & 0.009 & 0.026 & 0.009 & 0.040 & 0.021 & 0.007 & 0.043 & 0.222 & 0.006 \\
\hline population growth & - & - & 0.398 & 0.066 & 0.398 & 0.069 & 0.214 & 0.093 & 0.049 & 0.098 & 0.124 & 0.061 \\
\hline year & - & - & -0.014 & 0.002 & -0.014 & 0.002 & -0.004 & 0.005 & 0.127 & 0.014 & 0.088 & 0.130 \\
\hline lag response & - & - & - & - & - & - & - & - & - & - & -0.050 & 0.027 \\
\hline adj. $R^{2}$ & 0.102 & & 0.219 & & 0.236 & & 0.288 & & 0.638 & & - & \\
\hline sargan test $\mathrm{p}$ value & - & & - & & - & & - & & - & & 0.322 & \\
\hline $\mathrm{AR}(1) \mathrm{p}$ value & - & & - & & - & & - & & - & & 0.214 & \\
\hline $\mathrm{AR}(2) \mathrm{p}$ value & - & & - & & - & & - & & - & & 0.071 & \\
\hline$n$ & 2474 & & 2474 & & 2474 & & 2474 & & 2376 & & 2215 & \\
\hline
\end{tabular}

Table A7: Panel estimates of the dose-response function with bootstrapped standard errors.

\begin{tabular}{c|cccccccccccc}
\hline dose & \multicolumn{2}{c}{ POLS (no covariates) } & POLS (covariates) & \multicolumn{2}{c}{ RE } & \multicolumn{3}{c}{ FE } & \multicolumn{3}{c}{ FD } & \multicolumn{4}{c}{ GMM } \\
& $\widehat{\mu}(d)$ & S.E. & $\widehat{\mu}(d)$ & S.E. & $\widehat{\mu}(d)$ & S.E. & $\widehat{\mu}(d)$ & S.E. & $\widehat{\mu}(d)$ & S.E. & $\widehat{\mu}(d)$ & S.E. \\
\hline 1.000 & 1.010 & $(0.001)$ & 1.016 & $(0.002)$ & 1.016 & $(0.001)$ & 1.020 & $(0.001)$ & 1.019 & $(0.002)$ & 1.018 & $(0.005)$ \\
1.008 & 1.020 & $(0.001)$ & 1.022 & $(0.001)$ & 1.022 & $(0.001)$ & 1.024 & $(0.001)$ & 1.023 & $(0.001)$ & 1.023 & $(0.003)$ \\
1.013 & 1.026 & $(0.001)$ & 1.026 & $(0.001)$ & 1.026 & $(0.001)$ & 1.027 & $(0.001)$ & 1.027 & $(0.001)$ & 1.026 & $(0.002)$ \\
1.018 & 1.031 & $(0.001)$ & 1.030 & $(0.001)$ & 1.030 & $(0.001)$ & 1.030 & $(0.001)$ & 1.029 & $(0.001)$ & 1.029 & $(0.002)$ \\
1.023 & 1.036 & $(0.001)$ & 1.034 & $(0.001)$ & 1.034 & $(0.001)$ & 1.032 & $(0.001)$ & 1.032 & $(0.001)$ & 1.032 & $(0.002)$ \\
1.028 & 1.040 & $(0.001)$ & 1.037 & $(0.002)$ & 1.037 & $(0.001)$ & 1.034 & $(0.001)$ & 1.035 & $(0.001)$ & 1.035 & $(0.003)$ \\
1.033 & 1.044 & $(0.001)$ & 1.040 & $(0.002)$ & 1.040 & $(0.002)$ & 1.037 & $(0.001)$ & 1.038 & $(0.002)$ & 1.038 & $(0.004)$ \\
1.038 & 1.047 & $(0.002)$ & 1.043 & $(0.002)$ & 1.043 & $(0.002)$ & 1.039 & $(0.002)$ & 1.040 & $(0.002)$ & 1.041 & $(0.005)$ \\
1.043 & 1.050 & $(0.002)$ & 1.045 & $(0.002)$ & 1.046 & $(0.002)$ & 1.041 & $(0.002)$ & 1.043 & $(0.002)$ & 1.043 & $(0.006)$ \\
1.048 & 1.052 & $(0.002)$ & 1.047 & $(0.002)$ & 1.048 & $(0.002)$ & 1.043 & $(0.002)$ & 1.045 & $(0.003)$ & 1.046 & $(0.008)$ \\
1.050 & 1.053 & $(0.002)$ & 1.049 & $(0.003)$ & 1.049 & $(0.003)$ & 1.044 & $(0.002)$ & 1.046 & $(0.003)$ & 1.047 & $(0.009)$ \\
\hline
\end{tabular}


Table A8: Panel average treatment effect estimates with bootstrapped standard errors.

\begin{tabular}{|c|c|c|c|c|c|c|c|c|c|c|c|c|}
\hline \multirow[t]{2}{*}{ dose } & \multicolumn{2}{|c|}{ POLS (no covariates) } & \multicolumn{2}{|c|}{ POLS (covariates) } & \multicolumn{2}{|c|}{$\mathrm{RE}$} & \multicolumn{2}{|c|}{ FE } & \multicolumn{2}{|c|}{ FD } & \multicolumn{2}{|c|}{ GMM } \\
\hline & $\widehat{\tau}(d)$ & S.E. & $\widehat{\tau}(d)$ & S.E. & $\widehat{\tau}(d)$ & S.E. & $\widehat{\tau}(d)$ & S.E. & $\widehat{\tau}(d)$ & S.E. & $\widehat{\tau}(d)$ & S.E. \\
\hline 1.000 & & & & & & & & & & & & \\
\hline 1.005 & 0.007 & $(0.000)$ & 0.004 & $(0.001)$ & 0.004 & $(0.001)$ & 0.003 & $(0.001)$ & 0.003 & $(0.001)$ & 0.003 & $(0.002)$ \\
\hline 1.010 & 0.013 & $(0.001)$ & 0.009 & $(0.001)$ & 0.009 & $(0.001)$ & 0.006 & $(0.001)$ & 0.006 & $(0.001)$ & 0.006 & $(0.004)$ \\
\hline 1.015 & 0.019 & $(0.001)$ & 0.012 & $(0.002)$ & 0.013 & $(0.001)$ & 0.008 & $(0.00$ & 0.009 & $(0.002)$ & 0.009 & $(0.005)$ \\
\hline 1.020 & 0.024 & $(0.001)$ & 0.016 & $(0.002)$ & 0.016 & $(0.002)$ & 0.011 & $(0.002)$ & 0.012 & $(0.002)$ & 0.012 & $(0.007)$ \\
\hline 1.025 & 0.029 & $(0.002)$ & 0.020 & $(0.003)$ & 0.020 & $(0.002)$ & 0.013 & $(0.002)$ & 0.015 & $(0.003)$ & 0.015 & $(0.008)$ \\
\hline 1.030 & 0.033 & $(0.002)$ & 0.023 & $(0.003)$ & 0.023 & $(0.002)$ & 0.016 & $(0.002)$ & 0.018 & $(0.003)$ & 0.018 & $(0.009)$ \\
\hline 1.035 & 0.036 & $(0.002)$ & 0.026 & $(0.003)$ & 0.026 & $(0.00$ & 0.018 & $(0.00$ & 0.020 & $(0.003)$ & 0.021 & $(0.009)$ \\
\hline 1.040 & 0.039 & $(0.002)$ & 0.028 & $(0.003)$ & 0.029 & $(0.003)$ & 0.020 & $(0.003)$ & 0.023 & $(0.004)$ & 0.024 & $(0.010)$ \\
\hline 1.045 & 0.042 & $(0.002)$ & 0.031 & $(0.004)$ & 0.032 & $(0.003)$ & 0.022 & $(0.003)$ & 0.025 & $(0.004)$ & 0.026 & $(0.011)$ \\
\hline 1.050 & 0.043 & $(0.003)$ & 0.033 & $(0.004)$ & 0.034 & $(0.003)$ & 0.024 & $(0.003)$ & 0.028 & $(0.004)$ & 0.029 & $(0.012)$ \\
\hline
\end{tabular}

Table A9: Panel elasticity estimates with bootstrapped standard errors.

\begin{tabular}{|c|c|c|c|c|c|c|c|c|c|c|c|c|}
\hline \multirow[t]{2}{*}{ dose } & \multicolumn{2}{|c|}{ POLS (no covariates) } & \multicolumn{2}{|c|}{ POLS (covariates) } & \multicolumn{2}{|c|}{$\mathrm{RE}$} & \multicolumn{2}{|c|}{ FE } & \multicolumn{2}{|c|}{ FD } & \multicolumn{2}{|c|}{ GMM } \\
\hline & elas. & S.E. & elas. & S.E. & elas. & S.E. & elas. & S.E. & elas. & S.E. & elas. & S.E. \\
\hline 1.000 & & & & & & & & & & & & \\
\hline 1.005 & 1.374 & $(0.091)$ & 0.882 & $(0.133)$ & 0.894 & $(0.113)$ & 0.576 & $(0.121)$ & 0.643 & $(0.122)$ & 0.645 & $(0.427)$ \\
\hline 1.010 & 1.318 & $(0.084)$ & 0.857 & $(0.125)$ & 0.870 & $(0.106)$ & 0.566 & $(0.112)$ & 0.633 & $(0.117)$ & 0.637 & $(0.395)$ \\
\hline 1.015 & 1.262 & $(0.078)$ & 0.832 & $(0.118)$ & 0.846 & $(0.09$ & 0.555 & $(0.103)$ & 0.623 & $(0.1$ & 0.630 & $(0.364)$ \\
\hline 1.020 & 1.205 & $(0.072)$ & 0.807 & $(0.111)$ & 0.821 & $(0.092)$ & 0.545 & $(0.095)$ & 0.612 & $(0.107)$ & .622 & $(0.336)$ \\
\hline 1.025 & 1.149 & $(0.067)$ & 0.782 & $(0.104)$ & 0.797 & $(0.086)$ & 0.535 & $(0.087)$ & 0.602 & $(0.103)$ & 0.615 & $(0.310)$ \\
\hline 1.030 & 1.092 & $(0.062)$ & 0.757 & $(0.097)$ & 0.773 & $(0.081)$ & 0.525 & $(0.080)$ & 0.592 & $(0.100)$ & 0.607 & $(0.287)$ \\
\hline 1.035 & 1.036 & $(0.059)$ & 0.732 & $(0.090)$ & 0.749 & $(0.07$ & 0.515 & $(0.07$ & 0.582 & $(0.096)$ & 0.599 & $(0.269)$ \\
\hline 1.040 & 0.980 & $(0.056)$ & 0.707 & $(0.084)$ & 0.724 & $(0.071)$ & 0.505 & $(0.067)$ & 0.572 & $(0.094)$ & 0.592 & $(0.256)$ \\
\hline 1.045 & 0.923 & $(0.054)$ & 0.681 & $(0.078)$ & 0.700 & $(0.067)$ & 0.494 & $(0.063)$ & 0.562 & $(0.091)$ & 0.584 & $(0.249)$ \\
\hline 1.050 & 0.867 & $(0.054)$ & 0.656 & $(0.073)$ & 0.676 & $(0.065)$ & 0.484 & $(0.060)$ & 0.552 & $(0.090)$ & 0.577 & $(0.248)$ \\
\hline
\end{tabular}

\section{References}

Baltagi, B. H. (2002). An alternative derivation of mundlak's fixed effects results using system estimation. Econometric Theory 22, 1191-1194.

Cameron, A. C. and P. K. Trivedi (2005). Microeconometrics: methods and applications. Cambridge: Cambridge University Press.

Crump, R. K., V. J. Hotz, G. W. Imbens, and O. A. Mitnik (2009). Dealing with limited overlap in estimation of average treatment effects. Biometrika 96(1), 187-199.

Flores, C. A., A. Flores-Lagunes, A. Gonzalez, and T. C. Neumann (2012, February). Estimating the effects of length of exposure to instruction in a training program: The case of job corps. The Review of Economics and Statistics 94(1), 153-171.

Flores, C. A. and O. A. Mitnik (2009). Evaluating nonexperimental estimators for multiple treatments: Evidence from experimental data. IZA Discussion Papers 4451, Institute for the Study of Labor (IZA).

Hernán, M. A. and J. M. Robins (2012). Causal Inference. Monographs on Statistics and Applied Probability. London: CRC Press.

Hirano, K. and G. W. Imbens (2004). The propensity score with continuous treatments. In A. Gelman and X. L. Meng (Eds.), Applied Bayesian modeling and causal inference from incomplete data perspectives, pp. 73-84. New York: Wiley. 
Hymel, K. M., K. A. Small, and K. V. Dender (2010). Induced demand and rebound effects in road transport. Transportation Research Part B: Methodological 44(10), 1220-1241.

Imai, K. and D. A. Van Dyk (2004). Causal inference with general treatment regimes: generalizing the propensity score. Journal of the American Statistical Association 99(467), $854-866$.

Imbens, G. W. (1999). The role of the propensity score in estimating dose-response functions. NBER Working Paper $23 \%$.

Kluve, J., H. Schneider, A. Uhlendorff, and Z. Zhao (2012). Evaluating continuous training programmes by using the generalized propensity score. Journal of the Royal Statistical Society: Series A (Statistics in Society) 175(2), 587-617.

Melo, P., D. J. Graham, and S. Canavan (2013). Effects of road investments on productivity and induced travel demand: Evidence for U.S. urbanized areas. Transportation Research Record 2297, 163-171.

Mitnik, O. A. (2007). Intergenerational transmission of welfare dependency: The effects of length of exposure. Working Papers 0715, University of Miami, Department of Economics.

Myers, J. A., J. A. Rassen, J. J. Gagne, K. F. Huybrechts, S. Schneeweiss, K. J. Rothman, M. M. Joffe, and R. J. Glynn (2011). Effects of adjusting for instrumental variables on bias and precision of effect estimates. American Journal of Epidemiology 174(11), 1213-1222.

Neugebauer, R. (2004). Double Robust Estimation of Causal Parameters in Marginal Structural Models. Ph. D. thesis, University of California, Berkeley.

Neugebauer, R. and M. van der Laan (2005). Why prefer double robust estimators in causal inference? Journal of Statistical Planning and Inference 129(1-2), 405 - 426.

Pearl, J. (2010). On a class of bias-amplifying variables that endanger effect estimates. In Proceeding of the 25th Conference on Uncertainty in Artificial Intelligence (UAI 2010), Corvallis, pp. 425-432. Association for Uncertainty in Artificial Intelligence.

Ruppert, D., M. P. Wand, and R. J. Carroll (2003). Semiparametric regression. Cambridge: Cambridge University Press.

Wooldridge, J. M. (2010). Econometric analysis of cross section and panel data (2nd ed.). Cambridge, Massachusetts: MIT press. 